\title{
The prevalence of bipolar spectrum disorder in elderly patients with recurrent depression
}

\author{
This article was published in the following Dove Press journal: \\ Neuropsychiatric Disease and Treatment \\ 8 May 2014 \\ Number of times this article has been viewed
}

\author{
Chang-In Lee' \\ Young-Eun Jung' \\ Moon-Doo Kim' \\ Seong-Chul Hong ${ }^{2}$ \\ Won-Myong Bahk ${ }^{3}$ \\ Bo-Hyun Yoon ${ }^{4}$ \\ 'Department of Psychiatry, \\ ${ }^{2}$ Department of Preventive Medicine, \\ School of Medicine, Jeju National \\ University, Jeju, Republic of Korea; \\ ${ }^{3}$ Department of Psychiatry, Yeouido \\ St Mary's Hospital, College \\ of Medicine, The Catholic University \\ of Korea, Seoul, Republic of Korea; \\ ${ }^{4}$ Department of Psychiatry, Naju \\ National Hospital, Naju, Republic \\ of Korea
}

Purpose: Despite a growing body of knowledge on bipolar spectrum disorder (BSD), relatively little is known about the clinical characteristics of BSD in elderly people. We investigated the prevalence of BSD in elderly patients with recurrent depression.

Patients and methods: A total of 65 elderly outpatients ( $\geq 60$ years of age) who met the Diagnostic and Statistical Manual of Mental Disorders IV criteria for recurrent major depressive disorder participated in the study. BSD was diagnosed according to the criteria developed by Ghaemi et al and the Mood Disorder Questionnaire (MDQ) was used to assess bipolarity.

Results: Of 65 subjects, eleven (16.9\%) and 54 (83.1\%) were diagnosed with BSD and unipolar depression, respectively. A total of $32.3 \%(n=22)$ had a positive screen for bipolar disorder, and we found a significant association between the BSD criteria and the criteria for a positive MDQ $(P<0.001)$. Patients with BSD had a longer duration of illness $(P=0.040)$ and more prior depressive episodes $(P<0.001)$ than did those with unipolar depression. The BSD criteria of firstdegree relative with bipolar disorder $(P=0.030)$, antidepressant-induced hypomania $(P=0.034)$, hyperthymic personality $(P=0.001)$, and atypical depression $(P=0.030)$ were highly associated with MDQ-positive patients.

Conclusion: Our results indicate that many depressed elderly patients have bipolar-related illness; moreover, some features of the depression are associated with bipolarity.

Keywords: bipolarity, unipolar depression, MDQ, elderly

\section{Introduction}

Recent advances in psychiatry have increased our understanding of bipolar spectrum disorders and have further characterized the nature of conditions such as bipolar II disorder, the soft form of bipolar disorder, cyclothymic disorder, depressive mixed state, and the so-called bipolar spectrum disorder (BSD). ${ }^{1-5}$

The diagnostic boundaries of BSD are variable and require clarification and delineation. We defined BSD according to the criteria developed by Ghaemi et $\mathrm{al}^{6}$ thus, we excluded bipolar I and II disorder and focused on the softer expressions of bipolarity. BSD is associated with notable psychosocial disability and poor quality of life. ${ }^{7}$ However, patients with BSD typically experience subsyndromal symptoms of hypomania and no marked impairment; thus, they tend to seek treatment for depression, but not for mania or hypomania. Moreover, the depressive phases tend to be longer than the hypomanic and euthymic phases in patients with BSD. ${ }^{8}$ Thus, BSD is often under-referred, underdiagnosed, or misdiagnosed as another psychiatric disorder, such as unipolar depression. ${ }^{9}$ Smith et $\mathrm{al}^{10}$ found a high rate of BPD pathology in young adults with recurrent depression, and, of the patients in his sample, $47.1 \%$ met the diagnostic criteria for BSD. Similarly, elderly patients who experience recurrent depression may have BSD; however, little is known about the prevalence and the clinical features of the disorder in elderly people. 
The present study assessed the prevalence of bipolar spectrum in elderly patients with recurrent episodes of depression. BSD was diagnosed according to the criteria developed by Ghaemi et $\mathrm{al}^{6}$ and the Mood Disorder Questionnaire (MDQ), ${ }^{11,12}$ which is widely used as a screening instrument to detect bipolarity, was assessed.

\section{Material and methods}

We consecutively recruited outpatients aged 60 years and older who visited the Mood Disorder Clinic at Jeju National University Hospital (Jeju, Republic of Korea) between May 2010 and April 2011 and who met the Diagnostic and Statistical Manual of Mental Disorders Fourth Edition (DSM-IV) criteria for recurrent major depressive disorder (MDD). The diagnosis was made by a psychiatrist using the Mini-International Neuropsychiatric Interview semistructured interview. ${ }^{13}$ Exclusion criteria included a lifetime diagnosis of bipolar I disorder, psychotic disorder, severe personality disorder, any mental disorder resulting from a general medical condition, clinically evident dementia, and/ or medical issues likely to interfere with the patient's ability to participate in the study. A total of 65 outpatients who met the inclusion and exclusion criteria consented to participate in our study. The study was approved by the Jeju National University Hospital Review Board.

We used two approaches to identify BSD: the diagnostic criteria of Ghaemi et $\mathrm{al}^{6}$ and the MDQ score. ${ }^{11}$ We conducted a clinical interview with the patients using the eleven-item checklist of Ghaemi et al comprising criteria $\mathrm{C}$ (two items: first-degree relative with bipolar disorder; and antidepressant-induced hypomania) and criteria D (nine items: hyperthymic personality; at least three episodes of depression; brief major depressive episodes; atypical depression; psychotic depression; age of onset before 25 years; postpartum depression; antidepressant wear-off; and poor responses to more than two antidepressant trials). Patients were asked to complete a MDQ. The MDQ is a three-part self-report questionnaire that screens for a lifetime history of manic or hypomanic episodes: the first part contains 13 questions to assess symptoms related to mania or hypomania; the second part is a question about the co-occurrence of two or more symptoms; and the third part is a question about the extent to which symptoms have caused functional impairment. ${ }^{11,12}$ The Korean version of the MDQ has been standardized and its sensitivity (0.75) and specificity (0.69) for diagnosing bipolar disorder have been found to be adequate. ${ }^{14} \mathrm{We}$ defined a positive MDQ screen as a minimum of seven of 13 co-occurring symptoms resulting in moderate to severe functional impairment. ${ }^{14}$
The results are expressed as percentages for categorical variables and as means ( \pm standard deviation) for continuous variables. Group differences were calculated using independent $t$-tests for continuous variables and the chi-square test or Fisher's exact probability for categorical variables. The Kolmogorov-Smirnov test was used to examine differences in the distribution of age of onset of first episode between groups. All statistical analyses were conducted using the Statistical Package for the Social Sciences version 18.0 (SPSS Inc., Chicago, IL, USA) and an alpha level of 0.05 (two-tailed tests) was deemed to indicate statistical significance.

\section{Results}

Of the 65 subjects, eleven (16.9\%) were classified as BSD and $54(83.1 \%)$ as unipolar depression. A total of $32.3 \%(n=22)$ were MDQ positive and we found a significant association between the criteria for BSD and MDQ positivity $(P<0.001)$. Figure 1 shows the diagnostic agreement between MDQ results and the BSD C and D criteria.

Comparisons between BSD criteria positive/negative subjects and MDQ positive/negative subjects are presented in Table 1. The duration of illness was longer in patients with BSD than in those with unipolar depression $(P=0.040)$. We found that $81.8 \%$ of patients with BSD experienced more than three episodes of depression compared with $14.8 \%$ of patients with unipolar depression $(P<0.001)$. Figure 2 shows differences between MDQ-positive and MDQ-negative patients in relative frequencies of BSD criteria. Of the BSD criteria, first-degree relative with bipolar disorder $(P=0.030)$, antidepressant-induced hypomania $(P=0.034)$, hyperthymic personality $(P=0.001)$, and atypical depression $(P=0.030)$ were found to be highly correlated with MDQ-positive patients.

\section{Discussion}

Our results suggest that elderly patients who meet the DSM-IV criteria for recurrent MDD may have bipolar spectrum conditions. Overall, $16.9 \%$ of the patients satisfied the criteria for BSD developed by Ghaemi et al. ${ }^{6}$ These criteria give greater weight to family history and antidepressantinduced manic symptoms than other definitions, and they allow for the diagnosis of BSD in patients with MDD who have several signs of bipolarity without a spontaneous manic or a hypomanic episode. Unlike the BSD criteria, the self-rated MDQ screens for a lifetime history of manic or hypomanic episodes; that is, the primary focus is to identify the manic symptoms of bipolar I disorder. ${ }^{11} \mathrm{We}$ found that $32.3 \%$ of our patients screened positive for bipolar disorder on the MDQ. Moreover, we found a significant association between the 


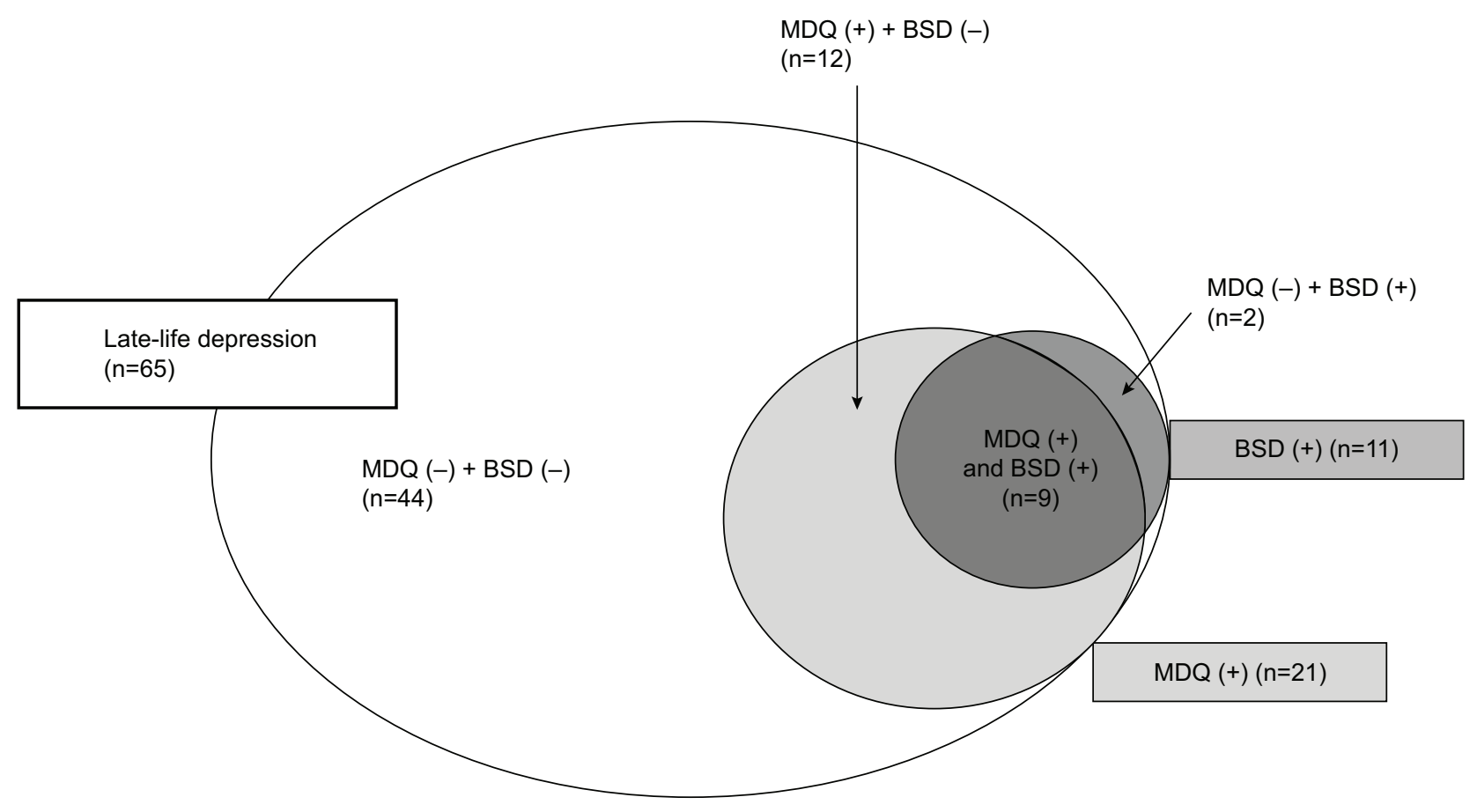

Figure I Prevalence of bipolar spectrum disorder according to the BSD criteria and MDQ.

Abbreviations: (+), positive; (-), negative; BSD, bipolar spectrum disorder; MDQ, Mood Disorder Questionnaire.

BSD criteria and those necessary for a positive MDQ screen. Our prevalence rate for BSD in elderly patients with recurrent depression was lower than that reported in a previous study in younger adults. ${ }^{10}$ The high prevalence of BSD in young individuals may be related to a shift to bipolar disorder in adolescents and young adults with depression..$^{15,16}$

In our study, patients who satisfied the criteria for BSD or were MDQ positive were significantly more likely to have a longer duration of illness, more prior depressive episodes, atypical depressive symptoms, family history for bipolar disorder, antidepressant-induced hypomania, and hyperthymic personality than patients with unipolar depression. Our results showing differences in clinical characteristics between elderly patients with BSD and unipolar disorder is an important finding and is consistent with a number of previous reports comparing the clinical characteristics of individuals with bipolar and unipolar depression. ${ }^{17-21} \mathrm{We}$ found no significant differences between the BSD and unipolar depression groups on psychotic features, earlier age of onset, postpartum depression, poor response to antidepressant trials, and suicidality. These findings may reflect our relatively small size and homogenous nature of the study sample. Furthermore, recall bias may have been a limiting factor in our study because elderly patients and their older family members were required to remember remote depressive episodes. Our study used self-report questionnaires, such as the MDQ; thus, considerable self-report bias may have influenced the results. A further concern is that we did

Table I Comparisons between BSD criteria positive/negative subjects and MDQ positive/negative subjects

\begin{tabular}{|c|c|c|c|c|c|c|}
\hline & \multicolumn{3}{|l|}{ BSD } & \multicolumn{3}{|l|}{ MDQ } \\
\hline & Positive $(n=I I)$ & Negative $(n=54)$ & $P$-value & Positive $(n=21)$ & Negative $(n=44)$ & $P$-value \\
\hline Age, years & $64.3 \pm 4.1$ & $63.8 \pm 3.4$ & 0.660 & $64.2 \pm 3.3$ & $63.7 \pm 3.6$ & 0.536 \\
\hline Female & $4(36.4 \%)$ & $35(64.8 \%)$ & 0.100 & $10(47.6 \%)$ & 29 (65.9\%) & 0.159 \\
\hline Age of onset of first episode, year & $45(24-65)$ & $55(23-67)$ & 0.295 & $54(24-65)$ & $55(23-67)$ & 0.870 \\
\hline Duration of illness, years & $21.3 \pm 12.6$ & $13.5 \pm 1 \mid .0$ & 0.040 & $17.7 \pm 12.9$ & $13.4 \pm 10.7$ & 0.161 \\
\hline At least 3 episodes of depression & $8(81.8 \%)$ & $9(14.8 \%)$ & $<0.00 \mathrm{I}$ & $9(42.9 \%)$ & $8(18.2 \%)$ & 0.034 \\
\hline Previous suicidal attempt & $2(18.2 \%)$ & $3(5.6 \%)$ & 0.196 & $2(9.5 \%)$ & $3(6.8 \%)$ & 0.655 \\
\hline Family history of mood disorder & $2(18.2 \%)$ & $4(7.4 \%)$ & 0.266 & $3(14.3 \%)$ & $3(6.8 \%)$ & 0.379 \\
\hline
\end{tabular}

Notes: Values are presented as number (\%), mean \pm standard deviation, or median (range). Statistical significance is designated by bold type. BSD group was classified by the explicit criteria developed by Ghaemi et al. ${ }^{6}$

Abbreviations: BSD, bipolar spectrum disorder; MDQ, Mood Disorder Questionnaire. 


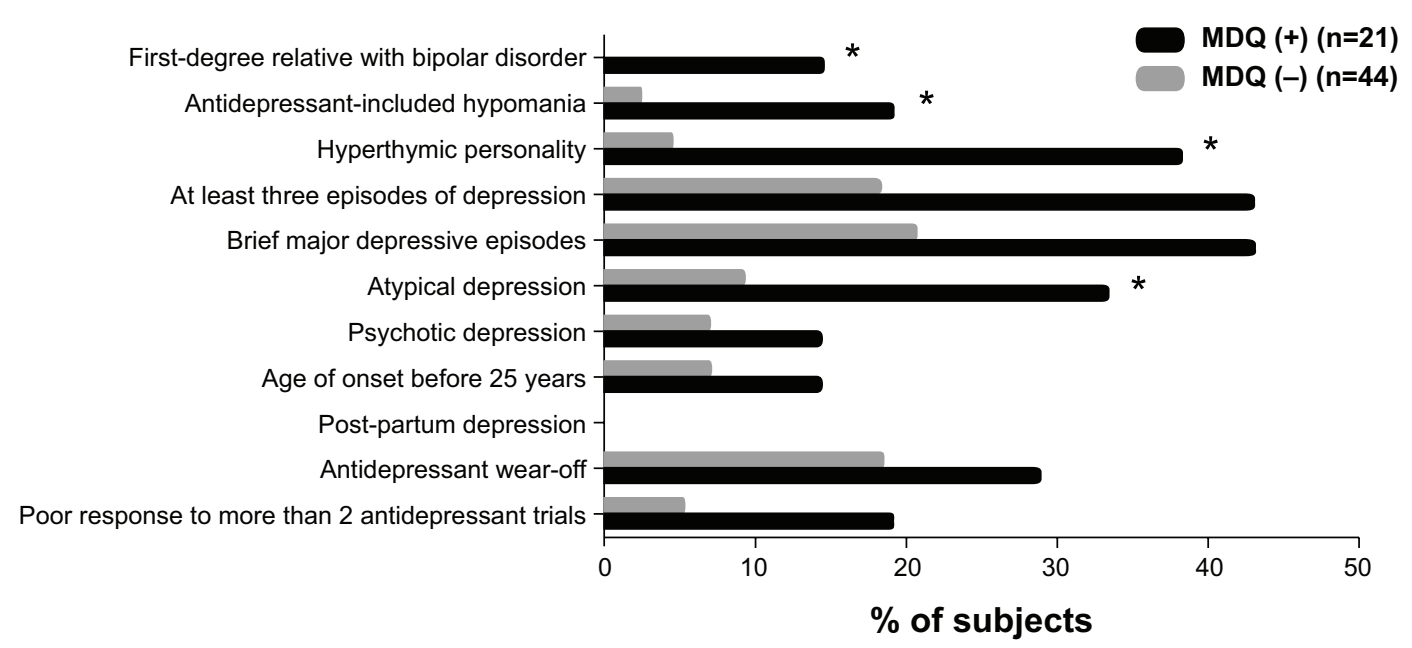

Figure 2 Comparisons between MDQ positive and MDQ negative patients in relative frequencies of BSD criteria (C and D). Note: *Indicates statistically significant.

Abbreviations: (+), positive; (-), negative; BSD, bipolar spectrum disorder; MDQ, Mood Disorder Questionnaire.

not evaluate the association between severity of depressive symptoms and self-assessment of hypomanic symptoms. Finally, strong evidence exists linking stress and emotional trauma with affect dysregulation; ${ }^{22,23}$ however, we did not evaluate various psychosocial factors that may have influenced mood instability, such as chronic environmental stress or traumatic experiences. Thus, we were not able to identify the proportion of patients in whom mood instability was caused by some form of stress rather than bipolarity.

\section{Conclusion}

Despite these limitations, the strength of our study is that we investigated the prevalence of BSD among elderly patients with DSM-IV recurrent MDD. Our results indicated that a considerable number of elderly patients with BSD have been underdiagnosed. Thus, clinicians should consider BSD as a differential diagnosis for late-life depression. Our findings are preliminary, and a larger prospective study is needed to confirm and generalize our results.

\section{Acknowledgment}

This work was supported by the research grant of Jeju National University in 2010.

\section{Disclosure}

The authors report no conflicts of interest in this work.

\section{References}

1. Akiskal HS, Pinto O. The evolving bipolar spectrum. Prototypes I, II, III, and IV. Psychiatr Clin North Am. 1999;22:517-534.

2. Akiskal HS, Akiskal KK, Lancrenon S, et al. Validating the bipolar spectrum in the French National EPIDEP Study: overview of the phenomenology and relative prevalence of its clinical prototypes. $J$ Affect Disord. 2006;96:197-205.
3. Hantouche EG, Angst J, Akiskal HS. Factor structure of hypomania: interrelationships with cyclothymia and the soft bipolar spectrum. $J$ Affect Disord. 2003;73:39-47.

4. Ghaemi SN, Ko JY, Goodwin FK. The bipolar spectrum and the antidepressant view of the world. J Psychiatr Pract. 2001;7:287-297.

5. Benazzi F. Bipolar II disorder: epidemiology, diagnosis and management. CNS Drugs. 2007;21:727-740.

6. Ghaemi SN, Ko JY, Goodwin FK. "Cade's disease" and beyond: misdiagnosis, antidepressant use, and a proposed definition for bipolar spectrum disorder. Can J Psychiatry. 2002;47:125-134.

7. Judd LL, Akiskal HS. The prevalence and disability of bipolar spectrum disorders in the US population: re-analysis of the ECA database taking into account subthreshold cases. J Affect Disord. 2002;73: 123-131.

8. Judd LL, Akiskal HS, Schettler PJ, et al. A prospective investigation of the natural history of the long-term weekly symptomatic status of bipolar II disorder. Arch Gen Psychiatry. 2003;60:261-269.

9. Dunner DL. Clinical consequences of under-recognized bipolar spectrum disorder. Bipolar Disord. 2003;5:456-463.

10. Smith DJ, Harrison N, Muir W, et al. The high prevalence of bipolar spectrum disorders in young adults with recurrent depression: toward an innovative diagnostic framework. $J$ Affect Disord. 2005;84:167-178.

11. Hirschfeld RM, Williams JB, Spitzer RL, et al. Development and validation of a screening instrument for bipolar spectrum disorder: the Mood Disorder Questionnaire. Am J Psychiatry. 2000;157: 1873-1875.

12. Zimmerman M, Galione JN. Screening for bipolar disorder with the Mood Disorders Questionnaire: a review. Harv Rev Psychiatry. 2011:19:219-228.

13. Sheehan DV, Lecrubier Y, Sheehan KH, et al. The Mini-International Neuropsychiatric Interview (M.I.N.I.): the development and validation of a structured diagnostic psychiatric interview for DSM-IV and ICD-10. J Clin Psychiatry. 1998;59 Suppl 20:22-33.

14. Jon DI, Hong N, Yoon BH, et al. Validity and reliability of the Korean version of the Mood Disorder Questionnaire. Compr Psychiatry. 2009;50:286-291.

15. Geller B, Zimerman B, Williams M, Delbello MP, Frazier J, Beringer L. Phenomenology of prepubertal and early adolescent bipolar disorder: examples of elated mood, grandiose behaviors, decreased need for sleep, racing thoughts and hypersexuality. J Child Adolesc Psychopharmacol. 2002;12:3-9.

16. Axelson D, Birmaher B, Strober M, et al. Phenomenology of children and adolescents with bipolar spectrum disorders. Arch Gen Psychiatry. 2006;63:1139-1148. 
17. Mitchell PB, Wilhelm K, Parker G, Austin MP, Rutgers P, Malhi GS. The clinical features of bipolar depression: a comparison with matched major depressive disorder patients. J Clin Psychiatry. 2001;62:212-216.

18. Solomon DA, Leon AC, Maser JD, et al. Distinguishing bipolar major depression from unipolar major depression with the screening assessment of depression-polarity (SAD-P). J Clin Psychiatry. 2006;67:434-442.

19. Benazzi F. Clinical differences between bipolar II depression and unipolar major depressive disorder: lack of an effect of age. J Affect Disord. 2003;75:191-195.

20. Perlis RH, Brown E, Baker RW, Nierenberg AA. Clinical features of bipolar depression versus major depressive disorder in large multicenter trials. Am J Psychiatry. 2006;163:225-231.
21. Benazzi F, Akiskal HS. How best to identify a bipolar-related subtype among major depressive patients without spontaneous hypomania: superiority of age at onset criterion over recurrence and polarity? $J$ Affect Disord. 2008;107:77-88.

22. Agid O, Kohn Y, Lerer B. Environmental stress and psychiatric illness. Biomed Pharmacother. 2000;54:135-141.

23. Zimmerman M, Ruggero CJ, Chelminski I, Young D. Psychiatric diagnoses in patients previously overdiagnosed with bipolar disorder. J Clin Psychiatry. 2010;71:26-31.

\section{Publish your work in this journal}

Neuropsychiatric Disease and Treatment is an international, peerreviewed journal of clinical therapeutics and pharmacology focusing on concise rapid reporting of clinical or pre-clinical studies on a range of neuropsychiatric and neurological disorders. This journal is indexed on PubMed Central, the 'PsycINFO' database and CAS, and is the official journal of The International Neuropsychiatric Association (INA). The manuscript management system is completely online and includes a very quick and fair peer-review system, which is all easy to use. Visit http://www.dovepress.com/testimonials.php to read real quotes from published authors.

Submit your manuscript here: http://www.dovepress.com/neuropsychiatric-disease-and-treatment-journal 